Marjolein H. Liedenbaum
A. H. de Vries
C. I. B. F. Gouw
A. F. van Rijn
S. Bipat
E. Dekker
J. Stoker

\section{CT colonography with minimal bowel preparation: evaluation of tagging quality, patient acceptance and diagnostic accuracy in two iodine-based preparation schemes}

Received: 27 March 2009

Revised: 1 July 2009

Accepted: 22 July 2009

Published online: 26 August 2009

(C) The Author(s) 2009.

This article is published with open access at Springerlink.com

M. H. Liedenbaum - A. H. de Vries . C. I. B. F. Gouw · S. Bipat · J. Stoker Department of Radiology,

Academic Medical Centre,

Amsterdam, The Netherlands

C. I. B. F. Gouw

Department of Radiology,

Gelre Hospitals,

Apeldoorn, The Netherlands

A. F. van Rijn · E. Dekker

Department of Gastroenterology and

Hepatology, Academic Medical Centre,

Amsterdam, The Netherlands

M. H. Liedenbaum $(\bowtie)$

Academic Medical Center,

G1-226, Meibergdreef 9,

1105 AZ Amsterdam, The Netherlands e-mail: M.H.Liedenbaum@amc.uva.nl

Tel.: +31-205663028

Fax: $+31-205669119$
Abstract Purpose: The aim of this study was to compare a 1-day with a 2-day iodine bowel preparation for CT colonography in a positive faecal occult blood test (FOBT) screening population. Materials and methods: One hundred consecutive patients underwent $\mathrm{CT}$ colonography and colonoscopy with segmental unblinding. The first 50 patients (group 1) ingested $7 * 50 \mathrm{ml}$ iodinated contrast starting 2 days before CT colonography. The latter 50 patients (group 2) ingested $4 * 50 \mathrm{ml}$ iodinated contrast starting 1 day before CT colonography. Per colonic segment measurements of residual stool attenuation and homogeneity were performed, and a subjective evaluation of tagging quality (grade 1-5) was done. Independently, two reviewers performed polyp and carcinoma detection. Results: The tagging density was 638 and $618 \mathrm{HU}$ $(\mathrm{p}=0.458)$ and homogeneity 91 and $86 \mathrm{HU}$ for groups 1 and 2, respectively $(\mathrm{p}=0.145)$. The tagging quality was graded 5 (excellent) in $90 \%$ of all segments in group 1 and $91 \%$ in group $2(p=0.749)$. Mean per-polyp sensitivity for lesions $\geq 10 \mathrm{~mm}$ was $86 \%$ in group 1 and $97 \%$ in group $2(p=0.355)$. Patient burden from diarrhoea significantly decreased for patients in group 2 . Conclusions: One-day preparation with meglumine ioxithalamate results in an improved patient acceptability compared with 2-day preparation and has a comparable, excellent image quality and good diagnostic performance.

Keywords CT colonography · Bowel preparation - Patient acceptance - Colorectal polyps . Colorectal carcinoma

\section{Introduction}

Computed tomography (CT) colonography (CT colonography) is an accurate method for detection of polyps and carcinomas in the colon and rectum, and can be considered a less burdensome examination compared with colonoscopy $[1,2]$. Recent studies have shown that faecal tagging only, i.e., without laxatives, is sufficient for bowel preparation [3-8]. Importantly, high diagnostic accuracy is found in studies that use such a limited bowel preparation for CT colonography [4, 6, 7]. Tagging for CT colonography can be performed with barium- or iodine-based contrast agents. Barium suspensions do not dissolve in liquids and are therefore more efficient at tagging only solid stools [8]. Non-ionic and ionic iodine-based agents dissolve in aqueous solutions, but can result in fluid shifts into the bowel lumen that generate soft stools and diarrhoea [9]. Zalis et al. compared barium and iodine contrast medium preparations and found improved discomfort scores and readability in the patient group that had ingested an iodine tagging agent [10]. Other studies also used iodine tagging in different amounts and often with use of additional laxatives $[3,11,12]$. A complicated scheme for ingestion of laxatives and tagging agents can however be difficult to follow for a patient, which can lead to incompliance [13]. Therefore, we aim to have a simple bowel preparation scheme, with ingestion of the oral contrast medium only. By increasing the amount of iodine 
contrast medium the stool will soften, which improves the image readability. Presently, no consensus exists on the amount of contrast medium and the number of preparation days needed for optimal tagging. An earlier study showed that a 1-day preparation with iodine contrast medium only might be sufficient to tag the faeces [12]. However, patient groups were small, which makes it difficult to draw conclusions regarding accuracy of polyp detection, and therefore a larger study is needed to substantiate these findings.

The aim of the present study was to compare the use of a 1-day versus a 2-day iodine-based bowel preparation for CT colonography in two patient groups by evaluating the patient acceptance, quality of the bowel preparation, homogeneity of tagged bowel contents and the accuracy of polyp detection.

\section{Materials and methods}

Between June 2006 and May 2007 a cohort of 10,000 patients between 50 and 75 years old received a faecal occult blood test (FOBT), either guaiac (Hemoccult II) or immunochemical (OC-Sensor), at home [14]. In total 302 patients who were willing to undergo $\mathrm{CT}$ colonography before colonoscopy were included in a CT colonography trial, which was previously reported [15]. Of these 302 patients, 100 consecutive patients that received a CT colonography in the Amsterdam region were included in this bowel preparation study. Exclusion criteria for FOBT positives were: persons unable to give informed consent, terminal illness, severe psychiatric symptoms, colonoscopy or an FOBT in the previous 2 years, examinations with radiation exposure in the last 12 months, previous contrast medium reaction, hyperthyroidism and pregnancy. The study was approved by the review board of the institution, and all participating patients gave informed consent.

\section{CT colonography examination}

\section{Bowel preparation}

All patients received a high-osmolar ionic monomer contrast medium (meglumine-ioxithalamate, $300 \mathrm{mg} \mathrm{I} / \mathrm{ml}$, Telebrix Gastro; Guerbert, Cedex, France). The first 50 consecutive patients received in total $350 \mathrm{ml}$ Telebrix (preparation 1, 2-day preparation) and the following 50 consecutive patients received $200 \mathrm{ml}$ Telebrix (preparation 2, 1-day preparation). The contrast medium was given without any additional fluid, but patients were allowed to drink the bottles of Telebrix mixed with syrup or water, for example. Furthermore, they were instructed to drink additional glasses of water during the day. In addition, patients followed a low-fibre diet. In Table 1 both preparation schemes are displayed.

\section{CT colonography acquisition}

CT was performed on a 64-slice CT system (Brilliance, Philips Medical Systems, Best, The Netherlands) at a slice collimation of $64 \times 0.625 \mathrm{~mm}$, pitch 1.2 , slice thickness $0.9 \mathrm{~mm}$, rotation time $0.4 \mathrm{~s}$, a tube voltage of $120 \mathrm{kV}$ and a reference $\mathrm{mAs}$ of 40 with dose modulation. A muscle relaxant, $20 \mathrm{mg}$ of butylscopolamine bromide (Buscopan; Boehringer-Ingelheim, Ingelheim, Germany), or, when contraindicated, $1 \mathrm{mg}$ of glucagon hydrochloride (Glucagen; Novo-Nordisk, Bagsvaerd, Denmark), was injected before insufflation of the colon. When these were both contraindicated, no muscle relaxant was injected. A balloon-tipped rectal catheter was inserted to insufflate approximately 31 of $\mathrm{CO} 2$ gas into the colon, using an automated insufflator (Bracco, $\mathrm{PROTOCO}_{2} 1$ insufflator, New York, USA).

\section{Evaluation of image quality}

Images were read on a Philips workstation (View Forum v 5.2, Philips Medical Systems) in 2D setting in a supine position only. The quality of tagging was assessed according to a rating scale [10] by two observers: MHL, a radiology research fellow, with previous experience of $350 \mathrm{CT}$ colonography readings, and CIBFG, a radiology fellow, with no previous experience with CT colonography reading, who received training on how to identify the appropriate bowel segment and how to evaluate homogeneity. The reading of the second observer was used to test observer agreement. The amount of faeces per colonic segment [16], the consistency of the residual faeces and the colonic distension were scored by the first observer on

Table 1 Bowel preparation scheme for the two preparations

\begin{tabular}{|c|c|c|c|}
\hline & Two days before CT colonography & One day before CT colonography & Day of CT colonography \\
\hline Preparation 1 & $\begin{array}{l}-3 * 50 \mathrm{ml} \text { Telebrix during each meal } \\
\text {-Low-fibre diet }\end{array}$ & $\begin{array}{l}1-3 * 50 \mathrm{ml} \text { Telebrix during each meal } \\
\text {-Low-fibre diet }\end{array}$ & $\begin{array}{l}-50 \mathrm{ml} \text { Telebrix } 1.5 \mathrm{~h} \text { before CT colonography } \\
\text {-Liquids before CT colonography }\end{array}$ \\
\hline Preparation 2 & -No diet restrictions & $\begin{array}{l}-3 * 50 \mathrm{ml} \text { Telebrix during each meal } \\
\text {-Low-fibre diet }\end{array}$ & $\begin{array}{l}-50 \mathrm{ml} \text { Telebrix } 1.5 \mathrm{~h} \text { before CT colonography } \\
\text {-Liquids before CT colonography }\end{array}$ \\
\hline
\end{tabular}


different rating scales (see Table 2). Assessments were performed after inclusion of all 100 patients. The patientstudy numbers were blinded so that the observers were not aware of the type of bowel preparation.

The homogeneity of the residual faeces was assessed by quantitative measurement by a second, independent observer [CIBFG, a radiology fellow who received training on how to perform region of interest (ROI) measurements]. Density (HU values) and homogeneity (SD values) of the tagged faeces were measured in all six colonic segments per patient. Slices were randomly selected by using a computer program (Windows Excel 2003, Microsoft) that produced six slice numbers for one CT colonography. These were correlated to the six colonic segments by the observer. When after the first randomisation a segment was not included, subsequent randomisations followed until all segments were measured. Per segment a specific ROI was drawn in the faeces to measure the attenuation (mean HU) and SD. See Fig. 1 for an example of an ROI drawing.

\section{Polyp detection at CT colonography}

Images were read by two experienced readers (AHdV, previous experience $400 \mathrm{CT}$ colonographies with colonoscopic verification and MHL, with 250) in a primary 2D setting, standard window level 1,500 and window width -250 , with $3 \mathrm{D}$ problem solving and a second read in 3D fly through. All lesions were marked and measured at 2D MPR. Of each position (prone and supine) the reading times were noted for reading primary $2 \mathrm{D}$ axial slices and the $3 \mathrm{D}$ fly-through second reading.

\section{Colonoscopy}

Within approximately 2 weeks (range: 1 to 22 days) of CT colonography, colonoscopy was performed. Bowel preparation for colonoscopy consisted of 41 polyethylene glycol electrolyte solution (KleanPrep; Helsinn Birex Pharmaceuticals, Dublin, Ireland) and a clear liquid diet starting the evening before colonoscopy. Experienced colonoscopists performed optical colonoscopy with a standard colonoscope (Olympus, Tokyo, Japan). Sedation, analgesics and/or a muscle-relaxant was used in all patients. According to the technique of segmental unblinding, the findings of the CT colonography were revealed to the colonoscopist after completing the examination of one segment. Polyp size was estimated by an opened biopsy forceps $(8 \mathrm{~mm})$ or by a linear measure probe (Olympus America). The histology of the lesion biopsies was classified as normal, hyperplastic, adenoma (type: serrated, tubular, tubulo-villous or villous and degree of dysplasia) or carcinoma according to the Vienna classification [17].

\section{Patient compliance and acceptance}

Patient experience of bowel preparation was evaluated by standardised questionnaires before the CT colonography and colonoscopy, and a mailed questionnaire 5 weeks after the colonoscopy. Patients were asked in the questionnaire before the CT colonography examination about their normal defecation pattern and how burdensome they found the overall CT colonography bowel preparation, and answered on a 5-point scale: $1=$ no discomfort, $2=$ mild, $3=$ moderate, $4=$ severe or $5=$ extremely

Table 2 Rating scales for the subjective scores on quality of bowel preparation and the colonic distension

\begin{tabular}{|c|c|}
\hline & Scale \\
\hline \multirow[t]{3}{*}{ Consistency of residual faeces } & 1. Liquid residual faeces \\
\hline & 2. Liquid and solid residual faeces \\
\hline & 3. Solid residual faeces \\
\hline \multirow[t]{4}{*}{ Amount of faeces per segment } & 1. $0 \%$ of the lumen filled with residual faeces \\
\hline & 2. $<25 \%$ of the lumen is filled with residual faeces \\
\hline & 3. $25-50 \%$ of the lumen is filled with residual faeces \\
\hline & 4. $\geq 50 \%$ is filled with residual faeces \\
\hline \multirow[t]{5}{*}{ Quality of tagging (average grade for one whole segment) } & 1. Non-interpretable images, untagged faeces and artefacts \\
\hline & 2. Poor interpretation, large amount of non-opacified faeces \\
\hline & 3. Moderate preparation, moderate amounts of non-opacified faeces \\
\hline & 4. Good preparation, small amounts of non-opacified faeces \\
\hline & 5. Excellent preparation, no non-opacified faeces \\
\hline \multirow[t]{4}{*}{ Colonic distension } & 1. Very poor distension; colon lumen cannot be identified \\
\hline & 2. Poorly distended; partly collapsed colon \\
\hline & $\begin{array}{l}\text { 3. Sufficient distension; suboptimal distended colon, but the colon } \\
\text { lumen is properly visible }\end{array}$ \\
\hline & 4. Well distended \\
\hline
\end{tabular}


Fig. 1 ROI box in a tagged fluid layer on a CT colonography

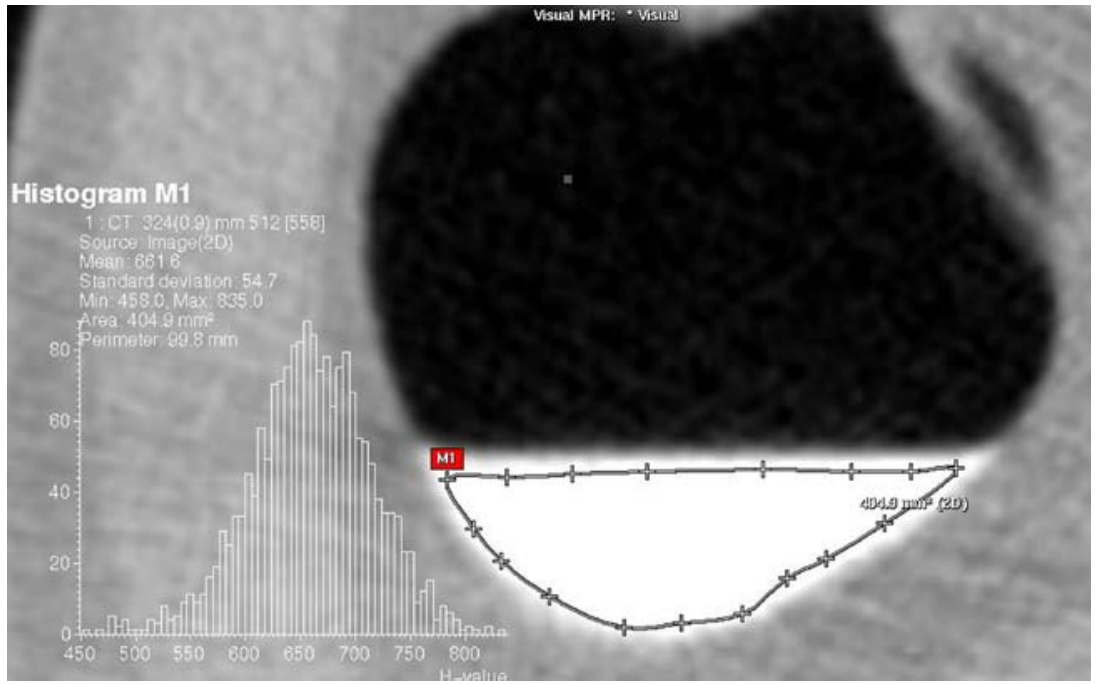

burdensome. Furthermore, they were asked if they had diarrhoea and how burdensome this was (assessed on a similar 5-point scale). Five weeks after the colonoscopy examination, patients were asked which examination or preparation they found most burdensome. The questionnaires had been used in previous studies on acceptance of CT colonography $[11,18]$.

\section{Statistical analysis}

No power calculation was performed, because we had no indication about the difference in homogeneity of the faeces in patients that would receive the 1-day versus the 2-day preparation. We estimated that a sample size of 50 patients per group should be sufficient to give insight into the quality of bowel preparation with an iodine tagging agent.

Age and sex distribution between the groups were tested using the Mann-Whitney and the chi-squared test respectively.

We estimated interobserver agreement for the quality of tagging by calculating weighted kappa statistics with corresponding $95 \%$ confidence intervals and by calculating the total number of concordant cases. The kappa values were interpreted as follows: $<0.20$ poor agreement; $0.21-0.40$, fair; $0.41-0.60$ moderate; $0.61-0.80$, good; $0.81-1.00$, excellent.

For the analysis of the amount of faecal residue, the quality of tagging, the colonic distension and the consistency of residual stool per segment, we applied ordinal regression analysis using generalised estimating equations (GEE) to revise the data clustering and dependency [19]. This was done because more than one segment per patient was used.

For comparison of the HU values (density) and SD values (homogeneity), the independent samples t-test was used to obtain means and standard deviation and to identify differences between the two groups. For overall analysis, a linear regression analysis was applied using GEE to revise the data clustering and dependency, and estimates of means with corresponding standard error were obtained.

For comparison of the reading times the independent samples t-test was used to identify differences between the two groups. For comparison of the different outcomes on the patient questionnaires, we performed an ordinal regression analysis.

CT colonography polyps were considered as true positive if the colonoscopy polyp was within one adjacent segment, if the estimated polyp size was within $50 \%$ of the colonoscopic measurement and if the morphology closely resembled the corresponding polyp seen on the videotaped colonoscopy. A per-polyp analysis was done resulting in a sensitivity and false-positive rate per observer and a per patient analysis resulting in a per-patient sensitivity and specificity. Results are given in two size categories: lesions of $6 \mathrm{~mm}$ and larger (medium) and lesions of $10 \mathrm{~mm}$ and larger (large). Lesions were categorised by size on the basis of the colonoscopic measurements, and the false positives were categorised according to the size measured at CT colonography. Only polyps with a possible pre-malignant histology (adenomatous or hyperplasia) and carcinomas were considered true lesions. Differences between the two preparation groups for per polyp sensitivity, per patient sensitivity and specificity were tested using the chi-squared test.

Statistical analyses were performed using MedCalc version 9.4.2.0 for Windows to calculate agreement, SAS version 8.02 for Windows (SAS Institute) to perform the GEE analyses, and all other analyses were done with SPSS version 15.0.1 for Windows (SPSS). For all analysis, a $p$ value of $<0.05$ indicated a significant difference between the two preparation groups. 


\section{Results}

Preparation group 1 consisted of 27 men and 23 women, group 2 of 30 men and 20 women $(p=0.545)$. Median age in group 1 was 58 years (range 50-72) and in group 2, 62 years $(50-75)(p=0.002)$. No adverse events occurred during the study. Nineteen patients were excluded; three patients were excluded because of hyperthyroidism, one because a previous allergic reaction to iodine intravenous contrast medium, nine had had a colonoscopy within 2 years, five had terminal illness and one had severe psychiatric symptoms.

\section{Evaluation of image quality CT colonography}

In group 1 the consistency of the residual faeces was liquid in 242 of a total of 285 segments (85\%); for group 2 this was 258 out of 285 segments (91\%). In both groups 15 segments contained no residual faeces. No significant differences were found in the consistency of the residual faeces among all segments of both groups $(p=0.122)$.

The subjective judgement on the homogeneity of the residual faeces was graded 5 (excellent) in $90 \%$ of all colon segments in the first preparation group and $91 \%$ in preparation group 2 . When the quality of tagging per segment was compared between the two preparation groups, no differences in homogeneity were found $(p=0.749)$. The results are summarised in Fig. 2. In Fig. 3 examples are given of different grades of preparation. Agreement in the quality of tagging between both observers in the caecum, ascending colon, transverse colon, descending colon, sigmoid and rectum was $88 \%$, $90 \%, 98 \%, 92 \%, 91 \%$ and $90 \%$, respectively. Weighted kappa statistics were $0.603,0.548,0.660,0.458,0.467$ and 0.469 , respectively, for these segments.

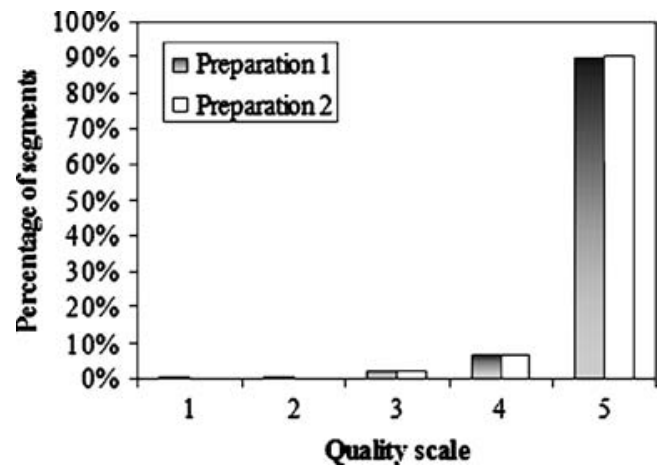

Fig. 2 Quality judgement of residual faeces for all colonic segments per preparation group $(\mathrm{p}=0.749) .1=$ non-interpretable images, untagged faeces and artefacts, $2=$ poor interpretation, large amount of non-opacified faeces, $3=$ moderate preparation, moderate amounts of non-opacified faeces, $4=$ good preparation, small amounts of non-opacified faeces, $5=$ excellent preparation no nonopacified faeces
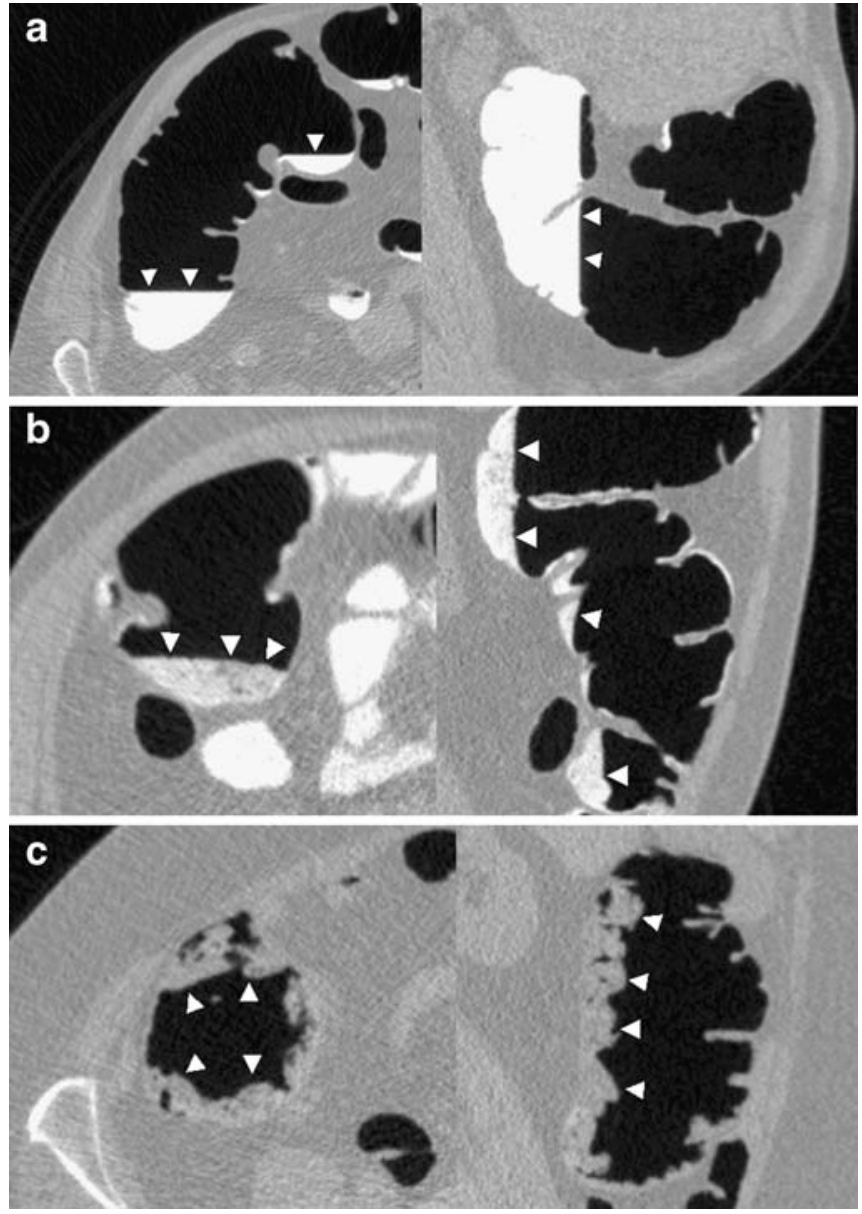

Fig. 3 Examples of different grades of preparation. At the left axial images and at the right the saggital images. White arrows indicate the faecal residues. a Excellent preparation (grade 5) in the caecum and ascending colon. b Moderate preparation (grade 3) in the caecum and ascending colon. $\mathbf{c}$ Non-interpretable preparation (grade 1) in the caecum and ascending colon

For the amount of residual faeces per segment and per preparation group, we found no significant difference between the two groups $(p=0.599)$. Seventy-two percent of the segments in group 1 and $69 \%$ of the segments in group 2 were filled with $0-25 \%$ of residual faeces.

Regarding the distension, the ascending colon received the highest grade (grade 4) for all segments in groups 1 and 2. A better distension of the sigmoid was found in preparation group 1 compared with group $2(\mathrm{p}=0.021)$. For the other segments no significant differences were found, and overall no difference existed between both groups $(p=0.200)$.

\section{Density and homogeneity of tagging}

The mean attenuation of the residual faeces in all segments was $638 \mathrm{HU}$ (SD 121) for preparation 1 and $618 \mathrm{HU}$ (SD $155)$ for preparation $2(p=0.456$; Table 3$)$. 
Table 3 Results of the density and homogeneity measurements per segment and per preparation group

\begin{tabular}{|c|c|c|c|c|c|c|}
\hline \multirow[t]{2}{*}{ Segment } & \multicolumn{3}{|l|}{ Density (HU) } & \multicolumn{3}{|c|}{ Homogeneity (SD) } \\
\hline & Preparation 1 & Preparation 2 & $\mathrm{p}$ values & Preparation 1 & Preparation 2 & $\mathrm{p}$ values \\
\hline Caecum & 620 & 599 & $\mathrm{p}=0.760$ & 81 & 77 & $\mathrm{p}=0.156$ \\
\hline Ascending & 624 & 591 & $\mathrm{p}=0.587$ & 81 & 78 & $\mathrm{p}=0.692$ \\
\hline Transverse & 683 & 670 & $\mathrm{p}=0.567$ & $105 \dagger$ & $91 \dagger$ & $\mathrm{p}=0.006$ \\
\hline Descending & 668 & 657 & $\mathrm{p}=0.198$ & $90 \dagger$ & $84 \dagger$ & $\mathrm{p}=0.036$ \\
\hline Sigmoid & 633 & 618 & $\mathrm{p}=0.105$ & 95 & 90 & $\mathrm{p}=0.384$ \\
\hline Rectum & 583 & 564 & $\mathrm{p}=0.072$ & 94 & 93 & $\mathrm{p}=0.178$ \\
\hline Total & 637 & 618 & $\mathrm{p}=0.456$ & 92 & 86 & $\mathrm{p}=0.148$ \\
\hline
\end{tabular}

$\dagger$ Indicates a significant difference

The homogeneity, the SD of the attenuation in the ROI, was $91 \mathrm{HU}$ (SD 22) for preparation group 1 and $86 \mathrm{HU}$ (SD 18) for preparation group $2(p=0.148$; see Table 3$)$. A better homogeneity in the transverse colon and descending colon was found in preparation group $2(\mathrm{p}=0.006$ and $\mathrm{p}=0.036$, respectively).

\section{Patient preference}

Most patients, $69 \%$ in group 1 and $67 \%$ in group 2, indicated that the consistency of their normal faeces was hard and dry; no differences were found in the consistency of normal stools $(p=0.062)$. The discomfort scores of the CT colonography bowel preparation of patients with preparations 1 and 2 are shown in Fig. 4. In group 1, 8\% (4/50) rated the CT colonography bowel preparation as extremely burdensome compared with $2 \%(1 / 49)$ in group $2(\mathrm{p}=0.388)$. In group 1 , six patients indicated that they did not take the total amount of 7 aliquots of $50 \mathrm{ml}$ of Telebrix (one patient ingested only 3 aliquots, three patients only 5 aliquots and one patient 6 aliquots). In group 2 all patients took the full amount, resulting in better compliance than in group $1(\mathrm{p}=0.013)$.

Diarrhoea was present in all patients of group 1; only two patients in group 2 reported that they did not have diarrhoea. In group 1, 26\% (13/50) rated the diarrhoea as

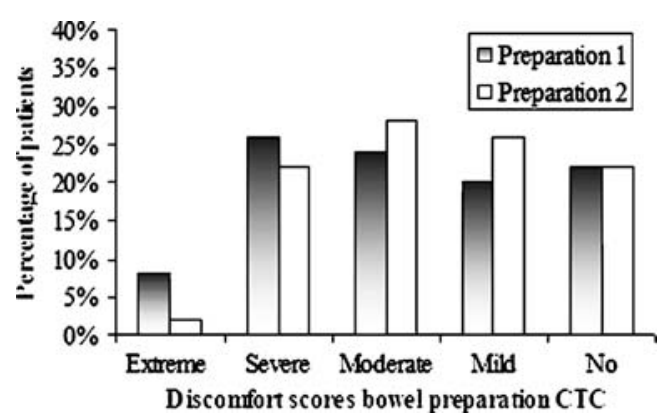

Fig. 4 Discomfort scores of the CT colonography bowel preparation per preparation group $(\mathrm{p}=0.388)$ extremely burdensome compared with $15 \%(7 / 47)$ in group 2 ( $\mathrm{p}=0.049$; see Fig. 5 for discomfort scores of diarrhoea). When comparing both preparations and examinations in both groups 1 and 2, 67\% found the colonoscopy bowel preparation the most burdensome aspect. See Fig. 6 for the results.

\section{Reading times}

Reading times of observers 1 and 2 were 15 min $46 \mathrm{~s}$ (SD $7 \mathrm{~min} 51 \mathrm{~s}$ ) and $15 \mathrm{~min} 06 \mathrm{~s}$ (SD $6 \mathrm{~min} 44 \mathrm{~s}$ ), respectively, in group 1 and $14 \min 50 \mathrm{~s}(\mathrm{SD} 3 \min 17 \mathrm{~s})$ and $12 \min 56 \mathrm{~s}$ (SD 6 min $16 \mathrm{~s})$ in group $2(\mathrm{p}=0.486$ for observer 1 and $\mathrm{p}=$ 0.057 for observer 2).

\section{Colonoscopy}

In the first group 33 lesions of $\geq 10 \mathrm{~mm}, 31$ adenomas and two carcinomas were identified at colonoscopy with segmental unblinding in 21 patients. In group 2, 33 lesions, of which 4 were carcinomas and 2 hyperplastic lesions, were found in 27 patients in this size category. For the category of lesions $\geq 6 \mathrm{~mm}, 61$ lesions (of which 4 were hyperplastic) in 34 patients and 68 lesions (of which 7 were

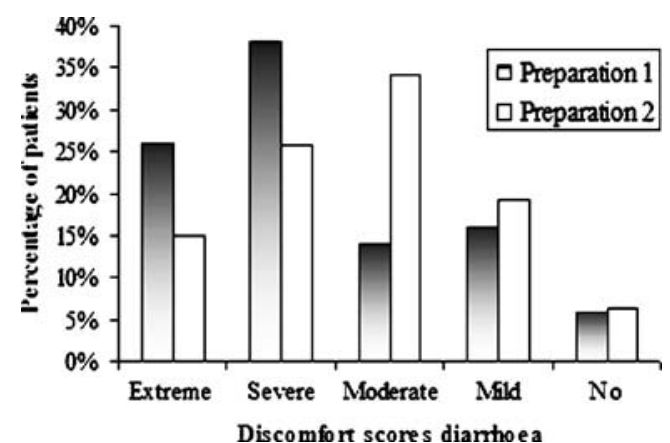

Fig. 5 Experience of diarrhoea in the two preparation groups $(\mathrm{p}=$ $0.049)$ 


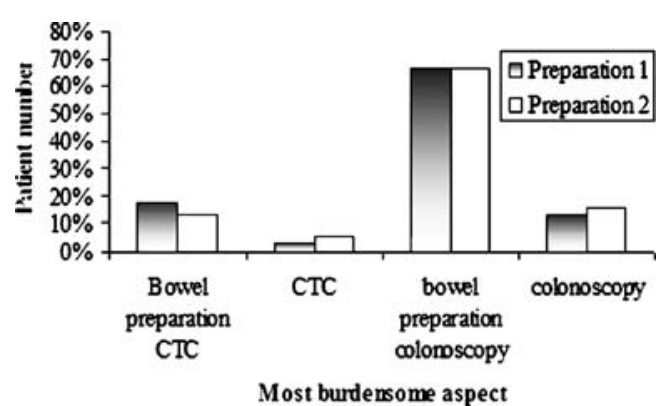

Fig. 6 The most burdensome aspect of the two examinations and bowel preparations in the two preparation groups $(\mathrm{p}=0.692)$

hyperplastic) in 40 patients were found in groups 1 and 2 , respectively.

\section{Polyp detection at CT colonography}

In Table 4 results are given for the per polyp sensitivity for reviewers 1 and 2 for both preparation groups and size categories. The mean sensitivity for lesions of $\geq 10 \mathrm{~mm}$ was $86 \%$ in group 1 and $97 \%$ for group $2(\mathrm{p}=0.355)$. The mean sensitivity for lesions of $\geq 6 \mathrm{~mm}$ was $76 \%$ in group 1 and $90 \%$ for group $2(\mathrm{p}=0.052)$. When considering adenomas and carcinomas $\geq 10 \mathrm{~mm}$ only, the mean sensitivity was also $86 \%$ in group 1 and $97 \%$ in group $2(p=0.360)$. For adenomas and carcinomas $\geq 6 \mathrm{~mm}$ the mean sensitivity was $78 \%$ in group 1 and $88 \%$ in group $2(p=0.157)$. Table 5 shows the results of the FP rate in both size categories and for both observers.

The per patient sensitivity is given in Table 6 . The mean sensitivity in group 1 was $90 \%$ for lesions $\geq 10 \mathrm{~mm}$ and in group $296 \%(\mathrm{p}=0.574)$. The sensitivity for lesions $\geq 6 \mathrm{~mm}$ in group 1 was $84 \%$, and in group 2 it was $98 \%$ with a significant difference for observer 1 . No differences in per patient specificity between the two preparation groups were found.

\section{Discussion}

In this study we compared a 1-day with a 2-day tagging only preparation scheme for CT colonography using a high- osmolar iodine contrast medium (meglumine-ioxithalamate) and a low-fibre diet. The most important results were that the image quality and polyp detection were comparable in the two groups. Tagging quality was given high scores in both groups, and ROI measurements of homogeneity and density were nearly equal; even a significantly better homogeneity in the transverse and descending colon in group 2 was found (thus, the 1-day preparation group). Furthermore, the burden of diarrhoea was decreased in the second group. In our institution nowadays the 1-day preparation scheme is used for routine clinical practice.

In this study we used a high-osmolar ionic contrast medium because this agent causes a fluid consistency of the faeces that mixes well with the iodine contrast medium and thus homogeneous tagging can be obtained [3]. The fluid consistency is useful because image readability improves when properly tagged fluid faeces shift if the patient turns from the supine to the prone position [10]. Furthermore, the high density and good homogeneity that were obtained are probably important for improved polyp detection. A previous study showed that with a tagged material density of $480 \mathrm{HU}$ or higher at a tube current of $40 \mathrm{mAs}$, the sensitivity for polyp detection is optimal [20]. When a cleansing algorithm is used to digitally subtract residual faeces a good homogeneity is necessary [7]. In both preparation groups in our study the density was more than $600 \mathrm{HU}$, and the homogeneity (SD) was less than $100 \mathrm{HU}$. Compared to earlier studies these are comparable results. In the study of Zalis et al. who tested two iodine preparations that started $48 \mathrm{~h}$ before scanning, tagging density was 500 to $550 \mathrm{HU}$, and the homogeneity (SD) was also less than $100 \mathrm{HU}$ [10] with corresponding high grades for subjective tagging scores. In a study of Taylor et al. the four used barium tagging preparations starting 1 or 2 days before scanning, and produced an average tagged fluid density of around $500 \mathrm{HU}$ [21].

We also used the grading system for evaluation of the tagging quality by Zalis et al. [10]. This is however very subjective, and therefore a second observer also performed this analysis in order to measure observer agreement. We found high scores for tagging quality in almost all segments in both groups. Agreement was more than $90 \%$ in five of six segments, and weighted kappa statistics were moderate to good, indicating there was a quite high agreement.

Table 4 Per polyp sensitivity for observers 1 and 2

Preparation $1 \quad$ Preparation 2 p values

Observer 1

Sensitivity $(95 \% \mathrm{CI})$

Observer 2

Sensitivity $(95 \% \mathrm{CI})$

\section{Lesions $\geq 10 \mathrm{~mm}$}

Lesions $\geq 6 \mathrm{~mm}$

Lesions $\geq 10 \mathrm{~mm}$

Lesions $\geq 6 \mathrm{~mm}$
$85 \%$ (73-97)

$75 \%(65-86)$

$88 \%$ (77-99)

$77 \%$ (66-88)
$97 \%(91-100)$

87\% (79-95)

$\mathrm{p}=0.197$

$\mathrm{p}=0.098$

97\% (91-100)

93\% (86-99)

$\mathrm{p}=0.355$

$\mathrm{p}=0.013$ 
Table 5 Total number of FPs per observer

\begin{tabular}{llll}
\hline & & Preparation 1 & Preparation 2 \\
\hline Observer 1 & & & \\
FP & Lesions $\geq 10 \mathrm{~mm}$ & 6 & 1 \\
& Lesions $\geq 6 \mathrm{~mm}$ & 12 & 5 \\
Observer 2 & & & \\
FP & Lesions $\geq 10 \mathrm{~mm}$ & 1 & 1 \\
& Lesions $\geq 6 \mathrm{~mm}$ & 3 & 5 \\
\hline
\end{tabular}

We found that both observers yielded high scores for per polyp and per patient sensitivity (per patient sensitivity $>90 \%$ for polyps $\geq 10 \mathrm{~mm}$ in both groups). Specificity per patient was also $>90 \%$ in both groups, except for observer 1 in preparation group one $(81 \%)$, because of a higher number of false positives. Iannacone et al. also showed high sensitivity and specificity for polyp detection in a prospective CT colonography study with a minimal bowel preparation that started 2 days prior to the examination (200 $\mathrm{ml}$ of oral iodinated contrast medium) [6]. Per-patient sensitivity was even $100 \%$ for polyps $\geq 10 \mathrm{~mm}$.

Furthermore, reading times of CT colonography are correlated with the adequacy of tagging and the amount of residual faeces. A previous study showed that reading times were faster in unprepped patients who had more segments with fluid instead of dry residue faeces [22]. In our study no difference was found in reading times between the two preparation groups, implying that readability of the examinations was equal.

Considering the patient acceptance, we found that patients of the 1-day preparation group found the diarrhoea less burdensome than the patients of the 2-day preparation group. This is probably caused by the duration of diarrhoea and the total amount of iodine contrast medium, which was $150 \mathrm{ml}$ less in the 1-day preparation group. Also compliance was better in the second group; all patients took the 4 aliquots of contrast medium in group 2 compared with only 44 of the 50 patients who took all 7 aliquots of contrast medium in group 1 . However, most patients $(67 \%)$ in both groups still found the cathartic colonoscopy preparation most burdensome, which was also found in previous studies that used iodine or barium tagging preparations [3, 10, 13].

In this study, a low-fibre diet was used with clear descriptions for the patients. It is assumed that this diet reduces bowel contents and results in better homogeneity of the tagged faeces. Fibres are water-holding and therefore will increase stool weight; however, colonic transit time is reduced because of stimulation of peristalsis [23, 24]. Most studies that use faecal tagging prescribe a low residue or low-fibre diet, but also studies exist that did not use a special diet $[4,7]$.

There are some potential limitations in this study. A first limitation is that only supine positions are assessed for image quality and homogeneity measurements. The residual faeces will change position, but will not change in consistency and tagging quality; therefore, the results on these aspects will not be different in supine and prone positions. Another disadvantage is that we used an ionic contrast medium that might cause anaphylactic reactions. However, serious adverse events with intravenous ionic contrast medium seldom appear, and no serious adverse reactions with oral iodine contrast medium are described [25]. No adverse events occurred in this study. Barium can be used as an alternative, but it tags mainly the solid residual faeces and not fluid [26]. Therefore, tagging is often inhomogeneous [10].

A third limitation is that no randomisation was used for the different bowel preparation schemes. This was due to the fact that patients were part of a larger FOBT screening trial in which we have changed the bowel preparation scheme after half of the patients were included [15]. Patients were equally distributed with regard to sex, and also the indication (positive screening FOBT) was similar, but the age was significantly different in the two groups. Age could be of influence on consistency of stools, because in the elderly an increased prevalence of constipation is seen, especially after the age of 65 years [27]. However, in this study we asked patients about their normal consistency of stools, and this was equal for both preparation groups. The consecutive inclusion of patients could also be detrimental because observers could have obtained increased reading skills in CT

Table 6 Per patient sensitivity per observer

\begin{tabular}{lllll}
\hline & & Preparation 1 & Preparation 2 & $\mathrm{p}$ values \\
\hline Observer 1 & & & & \\
Sensitivity $(95 \% \mathrm{CI})$ & Lesions $\geq 10 \mathrm{~mm}$ & $90 \%(78-100)$ & $96 \%(89-100)$ & $\mathrm{p}=0.574$ \\
& Lesions $\geq 6 \mathrm{~mm}$ & $82 \%(70-95)$ & $98 \%(93-100)$ & $\mathrm{p}=0.043$ \\
Specificity $(95 \% \mathrm{CI})$ & Lesions $\geq 10 \mathrm{~mm}$ & $93 \%(84-100)$ & $100 \%$ & $\mathrm{p}=0.497$ \\
& Lesions $\geq 6 \mathrm{~mm}$ & $81 \%(62-100)$ & $100 \%$ & $\mathrm{p}=0.262$ \\
Observer 2 & & & & \\
Sensitivity $(95 \% \mathrm{CI})$ & Lesions $\geq 10 \mathrm{~mm}$ & $90 \%(78-100)$ & $96 \%(89-100)$ & $\mathrm{p}=0.574$ \\
& Lesions $\geq 6 \mathrm{~mm}$ & $85 \%(73-97)$ & $98 \%(93-100)$ & $\mathrm{p}=0.088$ \\
Specificity $(95 \% \mathrm{CI})$ & Lesions $\geq 10 \mathrm{~mm}$ & $100 \%$ & $100 \%$ & $\mathrm{p}=1.00$ \\
& Lesions $\geq 6 \mathrm{~mm}$ & $94 \%(82-100)$ & $90 \%(71-100)$ & $\mathrm{p}=1.00$ \\
\hline
\end{tabular}


colonography. Indeed, we found an increased sensitivity per observer for lesions $\geq 6 \mathrm{~mm}$ in group 2 . However, this increased sensitivity was in the patient group that received the smallest amount of tagging agent. Furthermore, a steep learning curve over the course of 100 examinations is not to be expected in readers with a previous experience of more than $250 \mathrm{CT}$ colonographies.

\section{Conclusion}

A 1-day bowel scheme with meglumine-ioxithalamate and a low-fibre diet for CT colonography is better tolerated by patients than a 2-day bowel preparation scheme with the same tagging agent. Quality scores on tagging, consistency and amount of residual faeces and the homogeneity of the residual faeces were comparable in the two bowel preparation groups. Furthermore, the diagnostic accuracy of polyp detection remained high in the 1-day preparation group. Therefore, a 1-day preparation scheme with meglumine-ioxithalamate can be considered superior to a 2-day preparation scheme.

Acknowledgements Thanks to The Netherlands Organization for Health Research and Development (ZonMW: project number 62300036) for funding this project.

Open Access This article is distributed under the terms of the Creative Commons Attribution Noncommercial License which permits any noncommercial use, distribution, and reproduction in any medium, provided the original author(s) and source are credited.

\section{References}

1. Halligan S, Altman DG, Taylor SA et al (2005) CT colonography in the detection of colorectal polyps and cancer: systematic review, meta-analysis, and proposed minimum data set for study level reporting. Radiology 237:893904

2. Johnson CD, Chen MH, Toledano AY et al (2008) Accuracy of CT colonography for detection of large adenomas and cancers. N Engl J Med 359:12071217

3. Bielen D, Thomeer M, Vanbeckevoort D et al (2003) Dry preparation for virtual CT colonography with fecal tagging using water-soluble contrast medium: initial results. Eur Radiol $13: 453-458$

4. Callstrom MR, Johnson CD, Fletcher JG et al (2001) CT colonography without cathartic preparation: feasibility study. Radiology 219:693-698

5. Dachman AH, Dawson DO, Lefere P et al (2007) Comparison of routine and unprepped CT colonography augmented by low fiber diet and stool tagging: a pilot study. Abdom Imaging 32:96104

6. Iannaccone $\mathrm{R}$, Laghi A, Catalano $\mathrm{C}$ et al (2004) Computed tomographic colonography without cathartic preparation for the detection of colorectal polyps. Gastroenterology 127:1300 1311
7. Johnson KT, Carston MJ, Wentz RJ et al (2007) Development of a catharticfree colorectal cancer screening test using virtual colonoscopy: a feasibility study. AJR Am J Roentgenol 188: W29-W36

8. Lefere P, Gryspeerdt S, Baekelandt M et al (2004) Laxative-free CT colonography. AJR Am J Roentgenol 183:945-948

9. Katayama H, Yamaguchi K, Kozuka T et al (1990) Adverse reactions to ionic and nonionic contrast media. A report from the Japanese Committee on the Safety of Contrast Media. Radiology 175:621-628

10. Zalis ME, Perumpillichira JJ, Magee C et al (2006) Tagging-based, electronically cleansed CT colonography: evaluation of patient comfort and image readability. Radiology 239:149-159

11. Florie J, van Gelder RE, Schutter MP et al (2007) Feasibility study of computed tomography colonography using limited bowel preparation at normal and low-dose levels study. Eur Radiol 17:3112-3122

12. Neri E, Turini F, Cerri F et al (2008) CT colonography: same-day tagging regimen with iodixanol and reduced cathartic preparation. Abdom Imaging. doi:10.1007/s00261-008-9453-z

13. Jensch S, de Vries AH, Pot D et al (2008) Image quality and patient acceptance of four regimens with different amounts of mild laxatives for CT colonography. AJR Am J Roentgenol 191:158-167

14. Van Rossum LG, Van Rijn AF, Laheij RJ et al (2008) Random comparison of guaiac and immunochemical fecal occult blood tests for colorectal cancer in a screening population. Gastroenterology 135:82-90
15. Liedenbaum $\mathrm{MH}$, van Rijn AF, de Vries AH et al (2009) Using CT colonography as a triage technique after a positive Faecal Occult Blood Test in colorectal cancer screening. Gut. doi:10.1136/gut.2009.176867

16. Gryspeerdt S, Lefere P, Herman M, Deman R, Rutgeerts L, Ghillebert G et al (2005) CT colonography with fecal tagging after incomplete colonoscopy. Eur Radiol 15:1192-1202

17. Schlemper RJ, Riddell RH, Kato Y et al (2000) The Vienna classification of gastrointestinal epithelial neoplasia. Gut 47:251-255

18. van Gelder RE, Birnie E, Florie J et al (2004) CT colonography and colonoscopy: assessment of patient preference in a 5-week follow-up study. Radiology 233:328-337

19. Pepe MS (2003) The statistical evaluation of medical tests for classification and prediction: Oxford statistical science series 28. Oxford University Press, New York, pp 35-65

20. de Vries AH, Venema HW, Florie J et al (2008) Influence of tagged fecal material on detectability of colorectal polyps at CT: phantom study. AJR Am J Roentgenol 191:1101

21. Taylor SA, Slater A, Burling DN et al (2008) CT colonography: optimisation, diagnostic performance and patient acceptability of reduced-laxative regimens using barium-based faecal tagging. Eur Radiol 18:32-42 
22. Dachman AH, Dawson DO, Lefere $P$ et al (2006) Comparison of routine and unprepped CT colonography augmented by low fiber diet and stool tagging: a pilot study. Abdom Imaging 32:96-104

23. Chen HL, Haack VS, Janecky CW et al (1998) Mechanisms by which wheat bran and oat bran increase stool weight in humans. Am J Clin Nutr 68:711-719
24. Sunvold GD, Titgemeyer EC, Bourquin LD et al (1995) Alteration of the fiber and lipid components of a definedformula diet: effects on stool characteristics, nutrient digestibility, mineral balance, and energy metabolism in humans. Am J Clin Nutr 62:1252-1260

25. Tramer MR, von EE, Loubeyre $P$ et al (2006) Pharmacological prevention of serious anaphylactic reactions due to iodinated contrast media: systematic review. BMJ 333(7570):675
26. Lefere P, Gryspeerdt S, Marrannes J et al (2005) CT colonography after fecal tagging with a reduced cathartic cleansing and a reduced volume of barium. AJR Am J Roentgenol 184:1836-1842

27. Johanson JF, Sonnenberg A, Koch TR (1989) Clinical epidemiology of chronic constipation. J Clin Gastroenterol 11:525-536 